https://doi.org/10.46344/JBINO.2020.v09i06.23

\title{
HEPATOPROTECTIVE EFFECT OF ETHANOLIC RHIZOME EXTRACT OF NARDOSTACHYS JATAMANSI AGAINST ACUTE ALCOHOL-INDUCED LIVER INJURY IN WISTAR RATS
}

\author{
Ashutosh Kumar Yadav*, Reetu Yadav, Dr Arun Garg, Dr Talib Hussain
}

Department of Pharmacology,KR Manglam University Sohna Road Gurgaon.

\begin{abstract}
To investigate the hepatoprotective activity of ethanolic rhizome extract of Nardostachys jatamansi against acute alcohol-induced liver injury in Wistar rats. Ethanolic (50\%) extract of Nardostachys jatamansi (NJE 400 and $800 \mathrm{mg} / \mathrm{kg}$ body weight) was administered daily for eight days in experimental animals. To develop hepatotoxicity, animals were administered orally with alcohol $12 \mathrm{ml} / \mathrm{kg}$ at $2 \mathrm{~h}$ after the doses of NJE everyday for eight consecutive days except the normal group. The hepatoprotective activity was assessed using various biochemical parameters like serum aspartate aminotransferase, serum alanine transaminase, alkaline phosphate, serum lactate dehydrogenase, albumin, and bilirubin level. The results of the study demonstrated that the treatment with NJE significantly $(P<0.05)$ and dose-dependently prevented alcohol-induced increase in serum levels of hepatic enzymes. The results of this study indicate that the ethanolic rhizome extract of Nardostachys jatamansi may prevent alcohol-induced liver injury.
\end{abstract}

Keywords: Nardostachys jatamansi; ethanol; antioxidant; antihepatotoxicity 


\section{Introduction}

The liver plays an important role in the metabolism and elimination of various exogenous and endogenous compounds. It is involved with almost all the biochemical pathways to growth, fight against the disease, nutrient supply, energy provision, and reproduction. Alcohol abuse is one of the main causes of liver disease worldwide and has become a social problem (1). Alcohol is estimated to cause about $20-30 \%$ of oesophageal cancer, liver cancer, cirrhosis of the liver, homicide, epileptic seizures, and motor vehicle accident worldwide. The liver is the target organ of alcohol toxicity and causes fatty change, hepatitis, and cirrhosis in the liver. The major toxic metabolites of ethanol are acetaldehyde and free radicals (2). Consumption of alcohol induces changes in an intestinal brush-border membrane, serum haematology, and electrolytes (3). The deficiency in enzymatic and nonenzymatic activity is associated with the alcoholic in liver disease (4).

Now a day, the use of herbal natural products has enhanced worldwide attention. Many herbal products are claimed to assist in a healthy lifestyle. Medicinally, herbal drugs have made a major contribution to the treatment of hepatotoxic activity (5). Traditionally rhizome of Nardostachys jatamansi is used as cardio protective nervous system disorder and hepatoprotective. Roots of the plant have anticonvulsant activity, antidepressant activity, cardio protective activity, and hypolipidemic activity. The whole plant have hypolipidemic activity.oil of this plant demonstrated as antifungal activity (6).

Since there is only one scientific evidence available on Nardostachys jatamansi for hepatoprotective activity but there is no evidence on ethanolinduced liver damage and also unavailable how many dose of this plant is more effective than standard drug Silymarin. Therefore the present study was designed to evaluate the hepatoprotective activity of Nardostachys jatamansi rhizome extract against acute alcohol-induced liver injury in Wistar rats.

\section{Materials and Methods \\ Collection of plant materials}

The rhizome of Nardostachys jatamansi was collected from New Delhi local market in the month of December 2012. The plant material was authenticated by Dr. H.B.Singh (Taxonomist), National Institute of Science Communication and Information Resources (NISCAIR), Pusa Campus, New Delhi-110012. A voucher specimen of Nardostachys jatamansi (NISCAIR/RHMD/CONSULT/-2012-

$13 / 2167 / 173)$ was deposited in the institute for further reference.

\section{Preparation of Extract}

2020 November Edition | www.jbino.com | Innovative Association 
The freshly collected rhizome of Nardostachys jatamansi was washed with distilled water to remove dirt and soil and shade dried in a ventilated place at room temperature. Dried plant material were cut into small pieces and reduced to coarse powder by mechanical grinder and defatted with petroleum ether 160 $80^{\circ} \mathrm{C}$ ) further extraction were carried out with $50 \%$ ethanol by cold percolation method to avoid damage due to heat. The extract was filtered and concentrated under reduced pressure $\left(40 \pm 1^{\circ} \mathrm{C}\right.$ ) by using rotavapor (Model noDB- 5546, Decibel Instruments, Chandigarh) to dryness to get a constant weight. The \% yield was found to be $8.75 \% \mathrm{w} / \mathrm{w}$. The Nardostachys jatamansi extract (NJE) was stored in $-20^{\circ} \mathrm{C}$ freezer and used for Pharmacological investigation.

\section{Animals}

Wistar rats (150-200 g) were group housed $(n=6)$ under a standard $12 \mathrm{~h}$ light/dark cycle and controlled conditions of temperature and humidity (25 $\left.\pm 2{ }^{\circ} \mathrm{C}, \quad 55-65 \%\right)$. Rats received standard rodent chow and water ad libitum. Rats were acclimatized to laboratory conditions for 7 days before carrying out the experiments. The study protocols were approved by the Institutional Animal Ethics Committee (IAEC) of Integral University, Faculty of Pharmacy, Lucknow, India (Reg. no. 1213/GO/ac/08/CPCSEA).

\section{Drugs and Chemicals}

Ethanol (Jiangsu Huaxi Ltd, China) and Silymarin (Sigma chemicals, USA) were used in present study. All other chemicals and other biochemical used in the experiments were of analytical grade from different firms.

\section{Experimental designs}

Group -l: Control $10.5 \%$ CMC $1 \mathrm{ml} / \mathrm{kg}$, p.o.)

Group -Il: Ethanol (50\% $12 \mathrm{ml} / \mathrm{kg})$

Group -III: NJE (400mg/kg, p.o.) + Ethanol (12g/kg, p.o.)

Group -IV: NJE (800mg/kg, p.o.) + Ethanol (12g/kg, p.o.)

Group -V: Silymarin (100mg/kg, p.o.) + Ethanol (12g/kg, p.o.)

Animals were divided into five groups of 6 animals each. The first group received $0.5 \% \mathrm{CMC} 1 \mathrm{ml} / \mathrm{kg}$. The group II received $50 \%$ ethanol $12 \mathrm{ml} / \mathrm{kg}$ for one week. The groups III, IV and $\mathrm{V}$ received $400 \mathrm{mg} / \mathrm{kg}$ and $800 \mathrm{mg} / \mathrm{kg}$ of NJE and silymarin (100 mg/kg p.o.) respectively once a day for eight days. All groups of animals were administered orally with ethanol $12 \mathrm{ml} / \mathrm{kg}$ at $2 \mathrm{~h}$ after the doses of NJE and Silymarin, respectively (7). At the end of the $8^{\text {th }}$ day treatment i.e. $24 \mathrm{~h}$ after the last dose of extract and standard drugs the rats were anaesthetized and blood was collected by retro-orbital plexus followed by heart puncture and allowed to clot. After blood withdrawal animal was sacrificed. The serum was separated by centrifugation at $3000 \mathrm{rpm}$ at $4^{\circ} \mathrm{C}$ for 20

2020 November Edition | www.jbino.com | Innovative Association 
minutes for the analysis of various biochemical parameters. The liver tissue was washed twice with ice cold saline, blotted, dried and then weighed. The relative liver weight was calculated as the percentage ratio of liver weight to the body weight. A small portion of the tissue was fixed in formalin for histological examination.

\section{Biochemical Evaluation in Serum}

Serum aspartate aminotransferase (AST), Serum alanine transaminase (ALT), Alkaline Phosphate (ALP), Serum lactate dehydrogenase (LDH), Total Cholesterol $(\mathrm{CHL})$ and Albumin (ALB) was determined by using standard kits from Merck, Mumbai, India. Bilurubin was estimated by using standard kits from (Medsource ozone Biomedical). All estimation was carried out using UV spectrophotometer (Shimadzu, india). The estimation procedure is obtained in detail from leaflets provided by the commercially available kits are as follows

\section{Phytochemical screening}

NJE was subjected to phytochemical screening (8) for the detection of various phyto-constituents.

\section{Statistical analysis}

The data were represented as mean \pm SEM. Analysis of variance test was followed by individual comparison by Newman-Keuls test using Graph Pad Prism Software (Version 3.0) for the determination of the level of significance. The results were considered significant at $P<0.05$.

\section{Results}

Effect of NJE on body weight, liver weight and relative liver weight against ethanol induced hepatotoxicity in rats

Table 1 shows the body weight (initial and final) and liver weights of control and experimental groups of animals. The control and experimental groups of rats did not show any significant change in final body weight.

The ethanol treatment (group-II) significantly increased the relative liver weight $(P<0.001 ; 5.39 \pm 0.51)$ when compared to the control group (3.19 \pm 0.31 ). Administration of 400 and 800 $\mathrm{mg} / \mathrm{kg}$ NJE (group III and IV; $3.85 \pm 0.39$ and $3.36 \pm 0.34)$ significantly reduced $(P<$ $0.001)$ the relative liver weight when compared ethanol group (5.39 \pm 0.51$)$, respectively. The animals treated with NJE alone $(400 \mathrm{mg} / \mathrm{kg})$ showed no significant change in the body weight and relative liver weight when compared to control group 1.

\section{Effect of extracts on ALT, AST and ALP}

As shown in Table 2 state that the ethanol induced group were significantly $(\mathrm{P}<0.001)$ increased AST, ALT and ALP were significantly increased when compared with control group. The administration of Silymarin $(100 \mathrm{mg} / \mathrm{kg})$ showed AST and ALT\&ALP) was significantly $(P<0.001)$ decreased when compared with ethanol group. NJE $(400$ and $800 \mathrm{mg} / \mathrm{kg}$ ) treated group showed AST, ALT and ALP were significantly 
$(\mathrm{P}<0.001)$ decreased when compared with ethanol group.

\section{Effect of extracts on LDH, CHL, Bilirubin Direct and Total}

As represented in Table 2 revealed that the LDH, CHL, Bilirubin Direct and Total were significantly $(P<0.001)$ increased in ethanol induced group when compared with control group. The Silymarin 1100 $\mathrm{mg} / \mathrm{kg}$ ) treated group showed that the $\mathrm{LDH}, \mathrm{CHL}$ Bilirubin direct and total were significantly $(P<0.001)$ decreased when compared with ethanol group. The NJE $(400$ and $800 \mathrm{mg} / \mathrm{kg}$ ) treated group showed the LDH, CHL, Bilirubin direct and total were significantly $(P<0.001)$ decreased when compared with ethanol group.

\section{Effect of extracts on ALB}

As revealed in per Table 2 state that the ALB level were significantly $(P<0.001)$ decreased in ethanol induced group when compared with control group. The Silymarin $(100 \mathrm{mg} / \mathrm{kg})$ treated group showed that the ALB were significantly $(P<0.001)$ increased when compared with ethanol group. The NJE $(400$ and $800 \mathrm{mg} / \mathrm{kg}$ ) treated group showed the ALB level were significantly $(P<0.001)$ increased when compared with ethanol group.

\section{Phytochemical screening}

Phytochemical screening of NJE indicated the presence of alkaloids, sesquiterpenes i.e. Jatamansone or valeranone, other sesquiterpenes- Alphapatcho-ulense, angelicin, $\beta$-eudesemo, $\beta$ atchoulense, $\beta$-sitosterol, calarene, elemol, jatamansin, jatamansinol, jatamansone, n-hexaco- sanyl, nhexacosane, oroselol, patchouli alcohol, valeranal, valeranone, nardostachnol, seychellene, seychelane and nardostachone.

\section{Discussion}

Liver disease due to alcohol consumption is a common cause of death in adults, medicinal support to alcohol-induced liver dysfunction is indeed very meager. In order to reflect the protective effect of Nardostachys jatamansi, we established the alcoholinduced acute liver injury model in experiment. In the present study, the ethanolic rhizome extract of Nardostachys jatamansi (NJE) was observed to exhibit hepatoprotective effect by using 50\% ethanol induced hepatotoxicity in Wistar rat.

Nardostachys jatamansi is an important medicinal plant which is used in traditional medicine to treat many diseases. Nardostachys jatamansi contains the presence of alkaloids, sesquiterpenes i.e. Jatamansone or valeranone, other sesquiterpenes- Alphapatcho-ulense, angelicin, $\beta$-eudesemo, $\beta$ atchoulense, $\beta$-sitosterol, calarene, elemol, jatamansin, jatamansinol, jatamansone, n-hexaco- sanyl, nhexacosane, oroselol, patchouli alcohol, valeranal, valeranone, nardostachnol, seychellene, seychelane and nardostachone (8). 
The increase in the liver weight may be due to hepatotrophy or hepatomegaly. It is attributed to the fact that alcohol consumption causes accumulation of lipids and proteins in hepatocytes with an impaired protein secretion by hepatocytes. Water is retained in the cytoplasm of hepatocytes leading to enhance the liver cells resulting in enlarged total liver mass and volume. This alcohol induced increase in total liver weight was prevented by treatment with Nardostachys jatamansi extract. Previous studies agree with our results $(4,9)$.

Increased in the level of activities of AST, ALT and ALP in the blood reflect the damage of liver hepatocytes and indirectly impairment of liver functions following APAP-induced hepatotoxicity. In Table 2, AST, ALT and ALP activities were significantly elevated $(P<0.001)$ after administration of ethanol. Pretreatment with 400 and $800 \mathrm{mg} / \mathrm{kg}$ of Nardostachys jatamansi extract significantly reduced the elevation of these enzymes $(\mathrm{P}<0.001)$. The reduction of liver enzymes was seen to be to the level of the control group and it was also similar to the level of group pretreated with Silymarin. One of the hallmark signs of hepatic injury or damage is apparent leakage of cellular enzymes into plasma. In addition, the extent and type of liver injury or damage can be accessed based on the presence or absence of specific enzymes in the blood stream (10). In general measurement of alanine aminotransferase, aspartate aminotransferase and alkaline phosphatase are commonly used as marker enzymes in accessing ethanol induced hepatotoxicity (4). In this study, hepatoprotective effect of Nardostachys jatamansi is evidenced by the improvement AST, ALT, ALP, ALB and bilirubin levels. Pretreatment with Nardostachys jatamansi extract suppresses ethanol induced AST, ALT, ALP, $\mathrm{LDH}, \mathrm{CHL}$, Bilirubin direct and total elevations. Previous studies have reported elevations of these biochemical parameter after ethanol treatment (4, 11).

The hepatoprotective activity of Nardostachys jatamansi may be ascribed to the presence of phytochemical constituents especially alkaloids and sesquiterpenes, which have been found in its extract by phytochemical investigation. Previous studies have demonstrated that some alkaloids and sesquiterpenes have hepatoprotective activity $(12,13)$. From the results it is clear that the ethanolic rhizome extract of Nardostachys jatamansi has shown dose dependent activity among which at the dose level of $400 \mathrm{mg} / \mathrm{kg}$, p.o. shows greater activity that is comparable with the control and standard groups.

\section{Conclusion}

These findings indicate that alkaloids and sesquiterpenes present in Nardostachys jatamansi probably inhibit ethanol uptake by tissues and exert their protective effects. The Nardostachys 
jatamansi exerts improvement in liver function by preventing the production of reactive oxygen species and enhancing the antioxidant defence system capacity. The hepatoprotective effects of the ethanolic rhizome extract of Nardostachys jatamansi may possess antioxidant activities and ameliorate hepatic function of hepatic injury induced by alcohol. Our present study suggests that the ethanolic rhizome extract of Nardostachys jatamansi may be an effective therapeutic agent in ethanol-induced liver injuries.

\section{References}

1. Kumar KE, Harsha K, Sudheer V. In vitro antioxidant activity and in vivo hepatoprotective activity of aqueous extract of Allium cepa bulb in ethanol induced liver damage in Wistar rats. Food Science and Human Wellness. 2013;2134):132-8.

2. Suzuki $Y$, Cherian MG. Effect of ethanol on brain metallothionein in transgenic mice. Alcoholism: Clinical and Experimental Research. 2000;24(3):315-21. 3. Bashir $M$, Javed MT. Effects of ethanol on brain and pancreas weights, serum sodium and potassium, and haematological parameters in quail (Coturnix coturnix japonica). Avian pathology. 2005;34(2):96-100.

4. Lodhi P, Tandan N, Singh N, Kumar D. Green tea extract ameliorates ethanol induced liver injury in Albino rats.
American Journal of Phytomedicine and Clinical Therapeutics. 2014;2:603-8.

5. Soni $M$, Mohanty $P$, Jaliwala $Y$. Hepatoprotective activity of fruits of Prunus domestica. Int J Pharm Biosci. $2011 ; 2(2): 439-53$.

6. Sahu $R$, Dhongade $H$, Pandey A, Sahu P, Sahu V, Patel D. Medicinal Properties of Nardostachys jatamansi (A Review). Oriental Journal of Chemistry. 2016;32(2):859-66.

7. Ahmad $M$, Yousuf $S$, Khan $M B$, Hoda MN, Ahmad AS, Ansari MA, et al. Attenuation by Nardostachys jatamansi of 6-hydroxydopamine-induced parkinsonism in rats: behavioral, neurochemical, immunohistochemical studies. Pharmacology Biochemistry and Behavior. 2006:83(1):150-60.

8. Jha SV, Bhagwat AM, Pandita NS. Pharmacognostic and Phytochemical studies on the rhizome of Nardostachys jatamansi DC. using different extracts. Pharmacognosy Journal. 2012;4(33):1622.

9. Arulmozhi $V$, Krishnaveni $M$, Karthishwaran K, Dhamodharan G, Mirunalini S. Antioxidant and antihyperlipidemic effect of Solanum nigrum fruit extract on the experimental model against chronic ethanol toxicity. Pharmacognosy magazine. 2010;6(21):42. 10. Abou Seif HS. Physiological changes due to hepatotoxicity and the protective role of some medicinal plants. Beni-Suef University Journal of Basic and Applied Sciences. 2016;5(2):134-46.

2020 November Edition | www.jbino.com | Innovative Association 
11. Du S-Y, Zhang Y-L, Bai R-X, Ai Z-L, Xie $B-S$, Yang $H-Y$. Lutein prevents alcoholinduced liver disease in rats by modulating oxidative stress and inflammation. International journal of clinical and experimental medicine. 2015;8(6):8785.

12. Asadi-Samani $M$, Kafash-Farkhad N, Azimi N, Fasihi A, Alinia-Ahandani E, Rafieian-Kopaei M. Medicinal plants with hepatoprotective activity in Iranian folk medicine. Asian Pacific Journal of Tropical Biomedicine. 2015;5(2):146-57.

13. Miyakoshi $M$, Yamaguchi $Y$, Takagaki R, Mizutani K, Kambara T, Ikeda $T$, et al. Hepatoprotective effect of sesquiterpenes in turmeric. Biofactors. 2004;21 (1-4):167-70. 
Table 1 Effect of NJE on body weight, liver weight and relative liver weight against ethanol induced hepatotoxicity in rats

\begin{tabular}{|l|l|c|c|c|c|}
\hline Groups & Treatment & $\begin{array}{l}\text { Initial body } \\
\text { weight }(\mathbf{g})\end{array}$ & $\begin{array}{l}\text { Final body weight } \\
\text { (g) }\end{array}$ & Liver weight (g) & $\begin{array}{l}\text { Relative liver wt. (liver } \\
\text { weight/100g b.w.) }\end{array}$ \\
\hline I & Control & $148 \pm 6.1$ & $152 \pm 7.4$ & $4.86 \pm 0.41$ & $3.19 \pm 0.31$ \\
\hline II & Ethanol (50\%) & $149 \pm 5.7$ & $147 \pm 8.6$ & $7.93 \pm 0.53^{\#}$ & $5.39 \pm 0.51^{\# \#}$ \\
\hline III & NJE 400mg/kg + ethanol & $152 \pm 5.2$ & $154 \pm 9.8$ & $5.94 \pm 0.67^{* *}$ & $3.85 \pm 0.39^{* * *}$ \\
\hline IV & NJE 800mg/kg + ethanol & $150 \pm 4.5$ & $153 \pm 9.5$ & $5.14 \pm 0.43^{* *}$ & $3.36 \pm 0.34^{* * * *}$ \\
\hline V & Silymarin + ethanol & $151 \pm 7.4$ & $156 \pm 7.2$ & $5.02 \pm 0.38^{* *}$ & $3.22 \pm 0.32^{* * * *}$ \\
\hline
\end{tabular}

Values are expressed as mean \pm SEM; P values: ${ }^{\#}<0.01,{ }^{\#}<0.001$ compared with respective control group I; P values: ${ }^{*}<0.05,{ }^{* *}<0.01$ and ${ }^{* * *}<0.001$ compared with group II (ethanol). 
Table 2 Effect of NJE on serum AST, ALT, ALP (U/L), Bilirubin level (mg/dl), Albumin (gm/dl), LDH (U/L), (mg/dl) and CHL (gm/dl) against ethanol induced hepatotoxicity in rats

\begin{tabular}{|l|l|l|l|l|l|l|l|l|}
\hline Groups & AST (U/L) & ALT (U/L) & ALP (U/L) & LDH (U/L) & CHL (mg/dl) & ALB(gm/dl) & \multicolumn{2}{l|}{ Bilirubin (mg/dl) } \\
\cline { 4 - 8 } & & & & & & Direct & Total \\
\hline Control & $90.56 \pm 11.54$ & $39.23 \pm 6.23$ & $67.32 \pm 8.12$ & $364.11 \pm 26.21$ & $35.87 \pm 5.21$ & $4.12 \pm 0.11$ & $0.59 \pm 0.12$ & $0.19 \pm 0.03$ \\
\hline Ethanol & $291.21 \pm 25.94^{\#}$ & $157.15 \pm 12.71^{\#}$ & $176.97 \pm 12.23^{\#}$ & $668.24 \pm 28.52^{\#}$ & $79.68 \pm 6.89^{\#}$ & $2.96 \pm 0.08^{\#}$ & $2.99 \pm 0.31^{\#}$ & $1.71 \pm 0.06^{\#}$ \\
\hline NJ 400 & $223.84 \pm 21.33^{* *}$ & $89.25 \pm 09.11^{* *}$ & $97.45 \pm 10.65^{* *}$ & $502.21 \pm 13.29^{* *}$ & $53.41 \pm 6.11^{* *}$ & $3.78 \pm 0.10^{* *}$ & $1.26 \pm 0.17^{* * *}$ & $0.97 \pm 0.04^{* *}$ \\
\hline NJ 800 & $119.12 \pm 13.11^{* * *}$ & $48.47 \pm 6.21^{* * *}$ & $73.87 \pm 6.54^{* * *}$ & $392.83 \pm 18.57^{* * *}$ & $40.33 \pm 2.89^{* * *}$ & $4.03 \pm 0.07^{* * *}$ & $0.79 \pm 0.13^{* * *}$ & $0.28 \pm 0.05^{* * *}$ \\
\hline Silymarin & $95.33 \pm 12.36^{* * *}$ & $40.17 \pm 6.09^{* * *}$ & $66.54 \pm 7.61^{* * *}$ & $373.77 \pm 17.01^{* * *}$ & $34.83 \pm 3.21^{* * *}$ & $4.14 \pm 0.05^{* * *}$ & $0.61 \pm 0.11^{* * *}$ & $0.20 \pm 0.03^{* * *}$ \\
\hline
\end{tabular}

Values are expressed as mean \pm SEM; P values: ${ }^{\#}<0.01,{ }^{\# \#}<0.001$ compared with respective control group I; P values: ${ }^{*}<0.05,{ }^{* *}<0.01$ and ${ }^{* * *}<0.001$ compared with group II (ethanol). 